\title{
Formation of bulk-laminated materials by localized deformation during ECAP of an AA6060 aluminum alloy
}

\author{
Martin Franz-Xaver Wagner ${ }^{1, *}$ and Philipp Frint ${ }^{1}$ \\ ${ }^{1}$ Materials Science Group, TU Chemnitz, 09107 Chemnitz, Germany
}

\begin{abstract}
This paper addresses localized plastic flow during equal-channel angular pressing (ECAP) of an AA6060 aluminum alloy. We observe an alternating formation of shear bands and matrix bands during ECAP that leads to pronounced strain partitioning without cracking. Local deformation is analyzed by considering the distortion of indents along flow lines in the center of a split billet. We estimate equivalent strains of about 3.6 inside the shear bands, whereas plastic deformation in the adjacent matrix bands is almost negligible. Microstructural analysis by SEM and STEM confirms that the shear bands exhibit typical features of severely plastically deformed microstructures at the onset of forming an ultrafine-grained microstructure. We further present statistics of band widths, and we discuss the roles material hardening as well as ECAP die geometry (in terms of the inner die radius) in facilitating the recurrent localized deformation that, in the absence of crack nucleation, leads to the production of an interesting and novel type of bulk-laminated materials by ECAP.
\end{abstract}

\section{Introduction}

Localization of deformation is a common phenomenon in materials science; examples include Lüders bands (in mild steels) [1], Portévin-Le Châtelier bands (e.g. in aluminum alloys) [2,3], or martensite bands related to the stress-induced phase transformation (e.g. in shape memory alloys [4-6]). In many cases, localized deformation is directly related to material failure. This is, for instance obvious, for adiabatic shear bands formed at high strain rates, where a locally increased amount of plastic deformation results in (locally) more pronounced energy dissipation, a (local) increase of temperature, and thus in (locally confined) thermal softening that in turn leads to an even more pronounced concentration of further deformation inside the forming shear band [7,8]. ECAP one of the most promising severe plastic deformation methods that allows to produce bulk ultrafine-grained (UFG) materials [9-11] - is also often limited by shear band formation accompanied by crack nucleation and growth that ultimately leads to billet fragmentation [1214].

In the light of these predominantly detrimental effects, here we consider a somewhat surprising and special situation where repeated shear localization actually results in the generation of a new type of UFG material with bulk lamellar structure. We have recently studied ECAP of an AA6060 aluminum alloy that has been cold-worked by extrusion at room temperature prior to ECAP, [15]. Interestingly, the material exhibits a pronounced and recurrent formation of shear bands, separated by much less deformed regions (which we refer to as matrix bands in the remainder of this paper). No damage occurs under the processing conditions used in our ECAP experiments. The resulting billets consist of alternating layers of shear and matrix bands that exhibit very different microstructural and mechanical properties, respectively. In this paper, we briefly present information on the initial material and on the ECA-pressing procedure. We analyze local deformation by considering the distortion of indents in a split billet, and we discuss the resulting microstructural features of shear vs. matrix bands. We furthermore study band widths and their distributions in the billets. Finally, we briefly discuss two potential factors that influence the recurrent formation of shear and matrix bands, material hardening capacity and ECAP die geometry.

\section{Material and experiments}

An AA6060 aluminum alloy of commercial purity was investigated. The material with the chemical composition $0.49 \mathrm{Mg}-0.45 \mathrm{Si}-0.16 \mathrm{Fe}-0.026 \mathrm{Mn}-0.003 \mathrm{Cu}-0.003 \mathrm{Zn}-$

(bal.)Al (in wt.-\%) was obtained in a cast condition. It was first homogenized at $530{ }^{\circ} \mathrm{C}$ for 12 hours followed by water quenching. Prior to ECA-processing, the material was processed by extrusion at room temperature, introducing an equivalent strain of approximately 1.3. In contrast to commercial extrusion, this processing step can be considered as a first part of the severe plastic deformat-

\footnotetext{
* Corresponding author: martin.wagner@mb.tu-chemnitz.de
} 

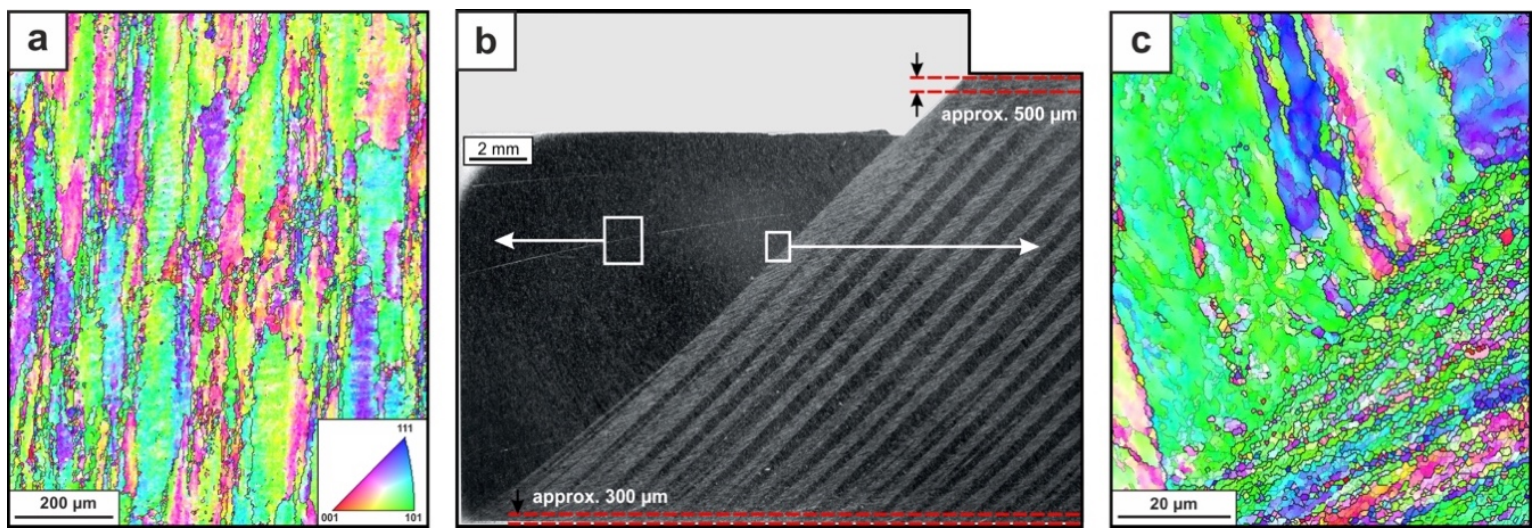

Fig. 1. Overview of the repeated formation of shear and matrix bands during ECAP of the cold-worked AA6060 aluminum alloy. (a) EBSD micrograph of the undeformed initial microstructure. (b) Low-magnification side-view on the end-piece of a billet after ECAP; shear bands (bright) and matrix bands (dark) form an alternating pattern. Band thickness is approximately constant from top to bottom of the billet, but in the very top/bottom layers (indicated by red dashed lines), the deformation appears to be more homogeneous. (c) EBSD micrograph of a region encompassing both undeformed material (upper left) and material that has passed the ECAP die's plastic deformation zone (lower right), consequently exhibiting pronounced grain refinement.

ion process (which is then followed by the main step during ECAP itself) since the pressing temperature is below the recrystallization temperature of the material.

ECAP billets with the dimensions of $15 \times 15 \times 50 \mathrm{~mm}^{3}$ were cut from the cold extruded material parallel to the extrusion direction. ECAP was conducted in a laboratory scale $90^{\circ}$-die (details of its design, which features movable walls to reduce friction between die and billet, are given in [16]) at room temperature and a pressing speed of $20 \mathrm{~mm} / \mathrm{min}$. Assuming an idealized shear zone geometry, as proposed by Iwahashi et al. [17], a single ECAP pass in a $90^{\circ}$-die is often estimated to result in an equivalent strain of 1.15. In order to further reduce friction during processing, a suitable wax-based lubricant (Bechem Beruforge 150D) was used [18]. A backpressure of $150 \mathrm{MPa}$ was applied to ensure that the deformation zone remained relatively thin and that no corner gap (i.e., an empty region near the outer corner of the die) occurred.

Microstructural analysis was performed by optical microscopy, electron backscatter diffraction (EBSD) and low voltage scanning transmission electron microscopy (STEM). The samples were prepared using a standard grinding and polishing procedure followed by either etching in a $4 \%$ sodium hydroxide solution (optical microscopy) or in colloidal silica (EBSD). STEM foils were prepared by electrolytic and ion polishing. Electron microscopy was conducted using a Zeiss Neon40EsB field emission microscope. During EBSD analysis, a suitable post-processing of the raw data was performed, including a slight clean-up, neighbor confidence index (CI) correlation and a grain CI standardization with a minimum $\mathrm{CI}$ of 0.1 .

\section{Strain partitioning during ECAP}

Fig. 1a shows an EBSD micrograph of the microstructure of the investigated aluminum alloy, taken from the end piece of a billet (shown as a macro scale micrograph in fig. 1b). This region has not passed through the plastic deformation zone in the ECAP die and therefore it is representative for the initial cold extruded material. The macroscopic view in fig. $1 \mathrm{~b}$ reveals an alternating arrangement of two different types of bands (both with approximately similar volume fractions), oriented parallel to the main shear plane in the ECAP die: shear bands that appear much brighter in the micrograph and dark matrix bands. Both types of bands exhibit an almost constant thickness from the top to the bottom of the billet; because of the geometrical constraints posed by the rigid exit channel of the ECAP die, the very top (width $\approx 500 \mu \mathrm{m}$ ) and bottom (width $\approx 300 \mu \mathrm{m}$ ) regions appear to be deformed more homogeneously as indicated in fig. $1 \mathrm{~b}$ (indeed, as discussed in greater detail in section 4, these regions are characterized by band branching). Figure 1c presents a magnified EBSD micrograph taken from the region surrounding the ECAP die's plastic deformation region (as highlighted in fig. 1b). An effective grain refinement is observed in the lower right hand-side of the plot (black lines indicate high angle grain boundaries), which represents a material volume that has been passed through the plastic deformation region. In contrast, coarse grains are observed on the upper left hand-side of the micrograph, corresponding to material that has not passed the plastic deformation region.

One focus of our previous study [15] was a detailed analysis of the local deformation path of material points passing through the plastic deformation region. We used a simple visio-plastic mapping technique by tracking indents that were prepared on a polished inner surface of a lengthwise split billet. An equidistant grid of hardness indents (Rockwell cone indenter) with a spacing of $400 \mu \mathrm{m}$ was prepared on the interior surface (see fig. 2). To prevent a plastic collapse of the indents during ECAP, a filling with an epoxy resin was applied prior to reassembling the billet. In order to ensure the characterization of the "steady-state" deformation condition, ECA-pressing was interrupted and the Lshaped billet (as shown schematically in fig. 2a) was 
extracted without further deformation by removing two walls of the die.
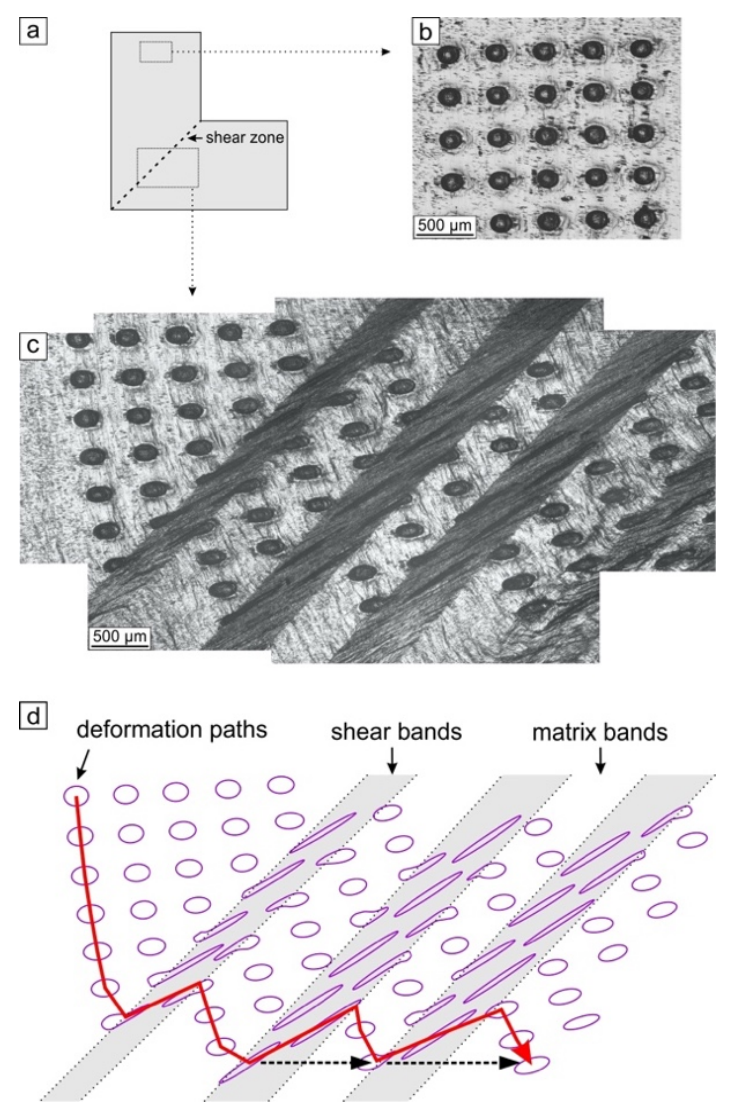

Fig. 2. (a) Schematic view of the L-shaped billet produced by interrupting an ECAP pass, showing the undeformed and deformed regions studied regions studied in greater detail. (b) Magnified view of the grid of indents in the undeformed region. (c) Grid of indents adjacent to the thin shear zone. Shear bands, characterized by pronounced plastic deformation, appear darker than the neighboring and much less deformed matrix bands. (d) Extracted positions and shapes of individual indents, determined from (c). By following the displacement of one line of indents, the deformation path through the ECAP die can be documented (indicated by the red line).

Fig. 2b shows a low magnification optical micrograph of the square grid of indents located in the entrance channel of the die (i.e. in the region that had not been subjected to plastic deformation in the shear zone of the die prior to interrupting the test). Despite the split billet having been put back together and subjected to a partial ECAP pass, the grid is undistorted, as are individual indents. This further indicates that our approach of analyzing grids in a split billet primarily records plastic deformation once it happens in the shear zone. Once material points are pushed close to the shear zone of the ECAP die, plastic deformation obviously commences, figs. $2 \mathrm{c}$ and $\mathrm{d}$. This is obvious both from the shapes of single indents, and from the representative deformation path highlighted by a red line in fig. $2 c$, located on the left of figs. $2 \mathrm{c}$ and $\mathrm{d}$. The deformation path already deviates significantly from the vertical orientation in the entrance channel of the die prior to reaching the shear zone itself (which is located close to the position of the left-most shear band in figs. 2c and d). In good agreement with analytical models of plastic flow in ECAP dies, like Beyerlein and Tomés fan-shape model $[19,20]$ or Tóth et al.'s super-elliptical flow line function approach [21,22], some initial plastic deformation is already accumulated along this part of the deformation path.

As discussed in detail in [15], a careful reconstruction of the shapes of the distorted indents (by fitting ellipses, fig. 2d) allows to analyze the deformation history along representative paths. From the considerably elongated indents inside the shear bands it is obvious that these regions have been severely deformed far exceeding the nominal shear strain that is typically introduced by a single pass of ECAP using a $90^{\circ}$ die. A simple analysis comparing indents in matrix and shear bands yields an estimated equivalent plastic strain due to this massive shear of about 3.6, [15]. In contrast, the matrix bands next to those severely sheared regions experience much less strain. Indeed, a comparison of the shapes of the corresponding indents with those in front of the shear zone reveals no differences, which is a strong indication that the matrix band regions have not been sheared further at all while passing the ECAP die's deformation zone.

It is particularly worth noting that the macroscopic deformation introduced into the billet must of course conform to the boundary conditions imposed by the ECAP die's geometry. The severe shear deformation inside the shear bands thus must be counterbalanced by the considerably reduced amount of plastic deformation in the matrix bands - a process that can be aptly referred to as recurrent strain partitioning. Considering the effective displacement vectors associated with deformation in a shear band an in an adjacent matrix band, this is obviously the case: the overall deformation results in horizontal material flow, i.e., parallel to the die's top and bottom in the exit channel (see black arrows in fig. 2d).

The low-resolution optical micrograph in fig. 3a, taken from the same perspective as in figs. 1 and 2, was further used to evaluate band widths, and to document band branching that occurs near the top and bottom surfaces of the billet. Fig. 3a confirms our estimate that shear and matrix bands exhibit similar width distributions; since they are arranged in an alternating pattern, their volume fractions are approximately $50 \%$. We highlight that band thickness does not vary significantly across the entire thickness of the billet, as shown by additional analyses from different cutting and viewing directions, [15]). By placing lines perpendicular to the interfaces between the bands, and employing a simple line intersection analysis, the shear band width distribution plot shown in fig. $3 \mathrm{~b}$ was determined. A closer inspection of the band structures in fig. 3a reveals that band branching occurs primarily near the bottom of the billet in a relatively wide zone, providing a gradual transition from the predominant reg- 

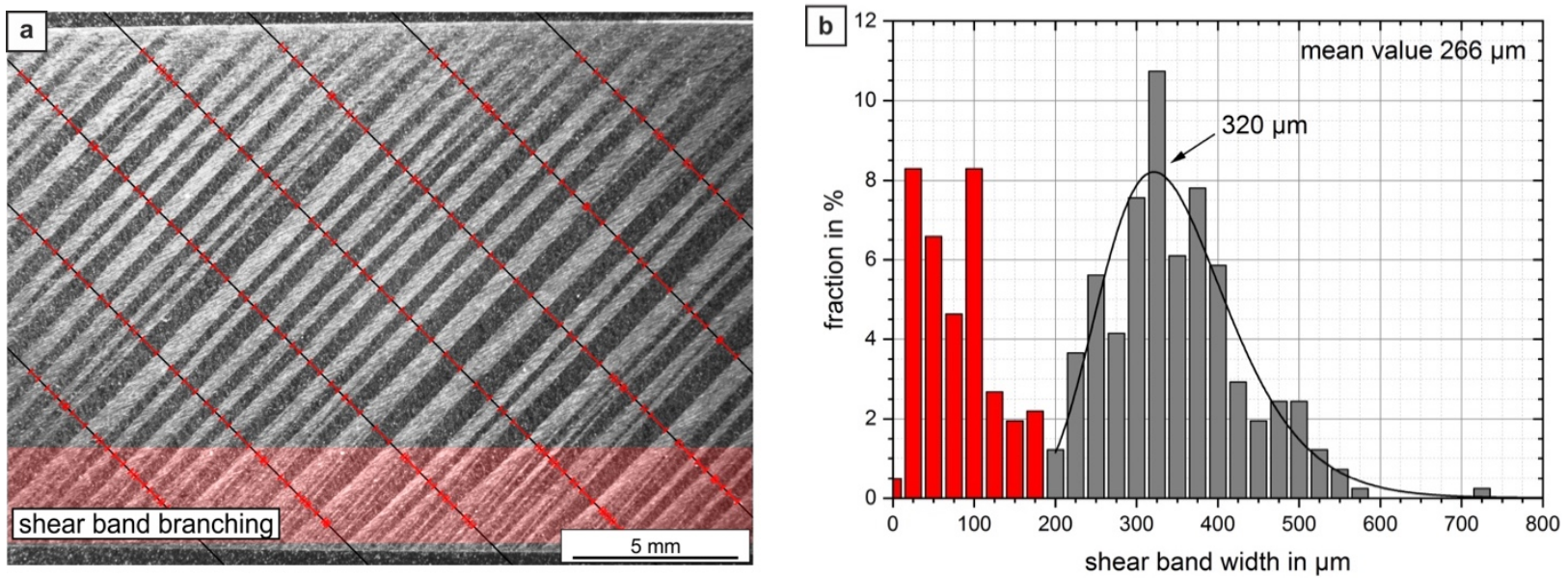

Fig. 3. (a) Shear and matrix bands, observed from the side of the billet exhibit a mostly constant thickness throughout the billet, but towards the top and (more pronounced) the bottom surface, band branching occurs (this transition region is highlighted in red). (b) Histogram of band width values determined from (a) using the line-intercept method. Shear band width exhibits a bimodal distribution with two peaks that can be directly related to the different regions in the billet (band widths in transition region: red; band width values in the main volume of the billet: grey).

ion of constant shear band width towards the outer material layers at the very bottom of the billet, which (due to the geometric constraints of the die) exhibits an apparently homogeneous deformation. Consequently, the shear band width distribution is bimodal with two distinct peaks that can be directly related to the different regions in the billet. In the region of band branching (also highlighted in red in fig. 3a), thin bands (red bars in the histogram, fig. 3b) with widths below $200 \mu \mathrm{m}$ are observed. Shear bands in the center part of the billet (grey bars in fig. 3b) approximately follow a normal distribution with a peak at a width of about $320 \mu \mathrm{m}$. Simple averaging of all band widths over the entire billet results in a mean value of $266 \mu \mathrm{m}$. To summarize, the statistical analysis demonstrates that the shear bands in this billet exhibit quite constant widths, typically on the order of several hundreds of microns.

\section{Microstructural features of shear and matrix bands}

Representative results of our detailed microstructural analysis are presented in fig. 4. EBSD was performed in the two different orientations shown schematically in Fig. 4a. The EBSD micrographs shown in figs. $3 \mathrm{~b}$ and $\mathrm{c}$ highlight representative microstructures for both, shear and matrix bands. Both images show a considerable grain refinement in the shear bands originating from a large amount of plastic deformation. In contrast, relatively large and much less deformed grains are clearly visible in the matrix bands. Even the elongated morphology of the grains formed by the previous room temperature extrusion can still be observed. It is moreover obvious that there still remains a pronounced crystallographic texture, especially in the matrix band regions. These findings agree well with

a
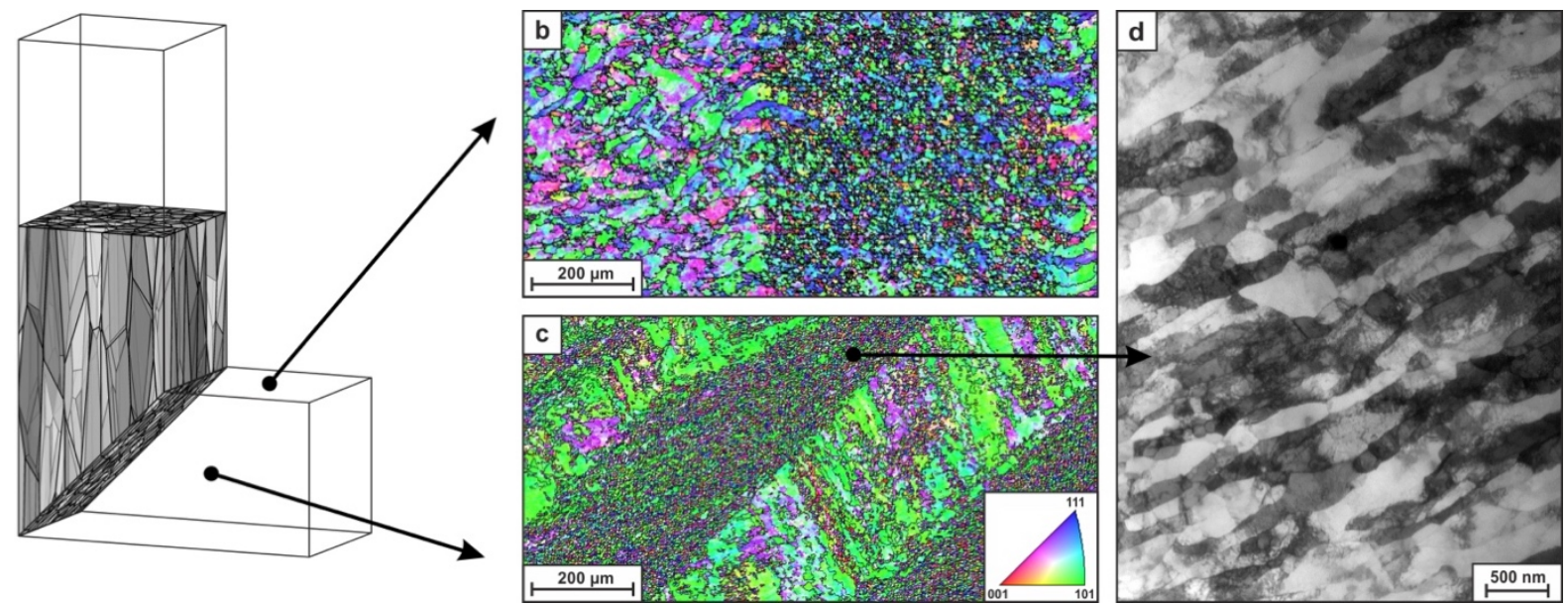

Fig. 4. Microstructural analysis of shear and matrix bands: (a) Schematic representation indicating the orientation of elongated grains (resulting from prior cold extrusion) and highlighting the viewing directions corresponding to the EBSD micrographs in (b) and (c). (d) The STEM image at higher magnification of the microstructure inside a single shear band clearly shows the formation of an UFG microstructure. 
the results of our strain analysis described above. Because of the very small grain size inside the shear bands and the physical limitations of EBSD measurements, additional low voltage scanning transmission electron microscopy (STEM) was performed, fig. $4 \mathrm{~d}$. These regions clearly are characterized by a fully developed UFG microstructure: The small, elongated grains have a length of approximately $500 \mathrm{~nm}$ and a width of about $200 \mathrm{~nm}$. The sharply defined boundaries between individual grains in the STEM image indicate substantial misorientations between grains - i.e., the boundaries have the characteristics of high angle grain boundaries. We emphasize that this observation is uncommon for $6 \mathrm{xxx}-$ series aluminum alloys deformed by a single ECAP pass; usually, multiple-pass ECAP, with a number of passes of about 4 (which corresponds to an equivalent strain of 4.4) is needed to generate the microstructural characteristics found in our shear bands.

\section{Effect of strain hardening and ECAP die geometry on recurrent strain partitioning}

The key scientific question raised by our experimental observations is why, and how, recurrent strain partitioning by shear banding during ECAP of the cold-worked AA6060 alloy occurs. Quite generally, unstable plastic flow may be expected when material hardening is insufficient to counterbalance softening effects (such as thermal softening, strain rate softening in materials with negative strain rate sensitivity, transformation softening, texture softening, or additional microstructural softening mechanisms), [23-26]. Indeed, shear band formation in aluminum subjected to high strains (but with shear banding on a much smaller length scale) has been investigated in detail, and discussed in relation to hardening behavior by Korbel and co-workers in the 1980s [27-30].

The role of different types and amounts of material hardening can also be evaluated in a straight-forward manner from a phenomenological, continuum mechanical point of view. Horn et al. recently analyzed our ECAP process by dedicated finite element simulations, carefully modeling the geometry of our die and varying the material behavior of the billet, [31]. The results of this study agree remarkably well with our experiments, particularly in terms of the resulting band widths. The numerical study demonstrated that the assumption of kinematic hardening is required to properly model shear band formation, whereas isotropic hardening only leads to homogeneous deformation in the simulations. Interestingly, when one numerically considers materials with different hardening rates and strain hardening capacities, distinct regions (associated with different types of homogeneous or inhomogeneous deformation during ECAP) can be identified in a representative stress-strain plot, fig. 5. One might expect from our general discussion of unstable plastic flow that a material with an overall low hardening rate and a low strain hardening capacity might be prone to localized deformation. However, such a material behavior (represented by flow curves in the lower region in fig. 5) is shown in [31] to only result in minor strain fluctuations; no distinct shear or matrix bands are formed and plastic strains only exhibit small gradients inside the billet. A more pronounced degree of strain hardening (i.e., stressstrain curves typically located inside the center region of the diagram in fig. 5), as expected, leads to stable plastic flow; no bands are formed and the deformation is mostly homogeneous (with some deviations, of course, in regions at the very top or bottom of the billet, where the ECAP die geometry affects the local stress states). Most importantly, the simulations reveal that materials with a pronounced initial strain hardening rate that quickly levels out after using up a certain amount of strain hardening capacity are prone to recurrent strain partitioning, forming welldefined shear and matrix bands as observed in our ECAP experiments.

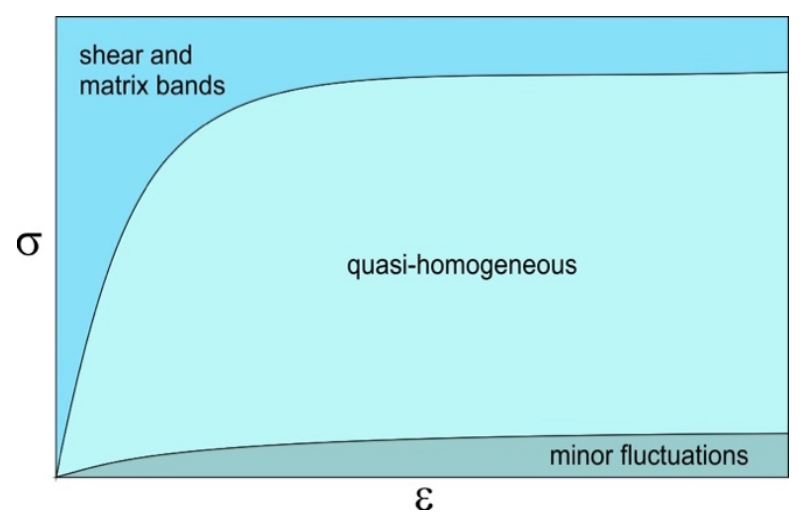

Fig. 5. Schematic comparison of different uniaxial flow curves as function of effective plastic strain, highlighting different material responses to ECAP, [31]: materials with a low hardening capacity exhibit only minor fluctuations in terms of equivalent plastic strain in only weakly defined bands. An increased hardening capacity suppresses this effect and leads to an apparently homogeneous deformation of the billet. In contrast, materials with a high initial hardening rate and a sufficiently large hardening capacity exhibit fully developed shear and matrix bands as observed in our experiments.

From a materials science point of view, it is furthermore critical to understand which microstructural conditions promote strain partitioning. Clearly, the coldworked microstructure prior to ECAP leads to the kind of mechanical behavior identified by the phenomenological finite element simulations, [31]. Moreover, we have shown in [15] that the material behavior during ECAP exhibits a pronounced orientation dependence: billets cut with different orientations with respect to the cold extrusion direction, when subjected to similar ECAP processing conditions, can exhibit either strain partitioning or conventional, homogeneous shear deformation. This obviously demonstrates that texture plays an important role. While a first tentative analysis of texture effects is discussed in [15], we acknowledge that further work is required to fully understand the effects of texture on shear band formation and unstable plastic flow in our AA6060 alloy during ECAP.

We also recorded the temporal evolution of the pressing force during ECA-pressing, analyzing distinct 
stress drops, [15]. Because of the optimized lubrication conditions (and because billets of the same alloy, but with different orientations, can exhibit homogeneous deformation under similar processing conditions), these stress drops cannot be related to stick-slip phenomena. Indeed, we clearly document that the stress drops can be directly associated with individual shearing events. The following qualitative scenario can therefore be used to describe recurrent strain partitioning mechanistically: as the billet is pressed through the die, stresses build up locally in the ECAP die's shear zone region (associated with strain hardening as discussed above) until they reach a critical value that leads to nucleation and rapid growth of a shear band through the billet's cross section. This dynamic process, and severe plastic deformation inside the shear band, result in mechanical unloading of the adjacent material, which follows the leading shear band through the deformation zone without additional massive shearing - until stresses gradually increase again and the entire process is repeated. This scenario implies a crucial role of local stress states, and this further suggests that specific details of the ECAP die geometry may have a significant effect on unstable plastic flow and shear band formation.

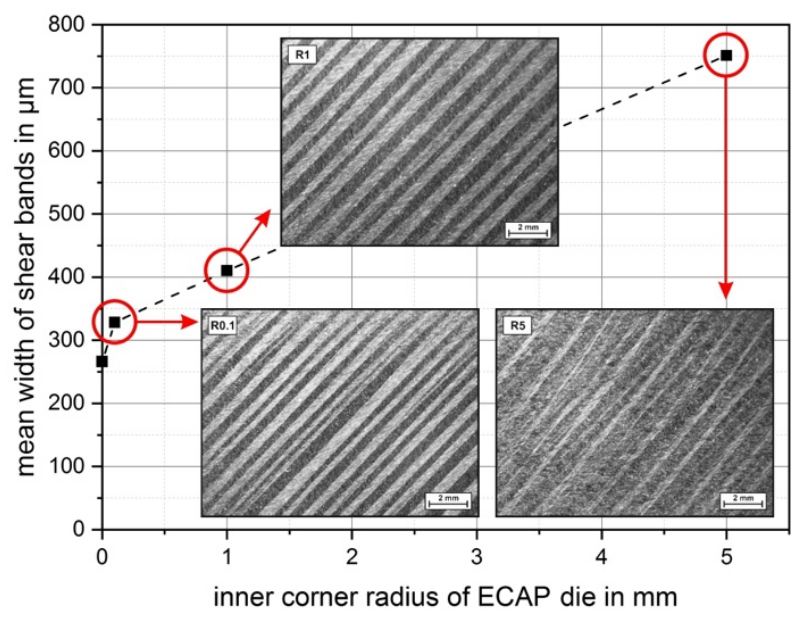

Fig. 6. Variation of mean shear band width as a function of the inner corner radius of the ECAP die. Insets show optical micrographs of shear and matrix bands (similar viewing direction as in figs. 1-3). With increasing corner radii, shear bands become wider and less well defined; for $\mathrm{R}=5 \mathrm{~mm}$, a transition towards an almost homogeneous type of deformation is observed.

To test this hypothesis, we modified a special detail of our ECAP die, its inner corner radius, where stress peaks are likely to occur during ECA-pressing, [31,32]. Fig. 6, where the average shear band width is plotted as a function of the inner corner radius, summarizes the results of this experimental parameter study. The micrographs presented as in-sets in fig. 6 further give an impression of the resulting shear and matrix band structures in the billets that were otherwise subjected to similar pressing conditions. Our earlier ECAP experiment was performed in the die with a nominally sharp inner corner radius $(\mathrm{R} \approx$ $0 \mathrm{~mm}$ ), which acts as a local stress raiser and leads to pronounced stress gradients in its vicinity. Increasing corner radii lead to reduced local stress peaks and less pronounced stress gradients. This obviously correlates with increasing (mean) shear band widths. An inner corner radius of $5 \mathrm{~mm}$ still results in recurrent strain partitioning; however, the shear bands are much less welldefined than in the cases involving smaller radii. While an in-depth analysis of local deformation in the different shear band populations produced using different die geometries is on-going work, it appears that the case of $\mathrm{R}=5 \mathrm{~mm}$ represents a transition state towards an almost homogeneous shear deformation. Clearly, our novel experimental results confirm a pronounced effect of ECAP die geometry, most likely related to the local stress state and its interaction with the nucleating shear bands, on the characteristic widths of shear and matrix bands. This observation opens an additional pathway that potentially allows to modify the structure of bulk laminated billets produced by ECAP of AA6060 by relatively simple engineering approaches, particularly ECAP die design.

\section{Summary and conclusions}

The experimental and numerical results presented in this paper address a novel phenomenon during ECAP of a cold-worked AA6060 alloy: strain partitioning by recurrent formation of shear bands and matrix bands. The resulting materials are characterized by alternating layers of these bands, with mean bands widths of several hundreds of microns, and can be considered as a type of bulk laminated material with partially UFG layers. Plastic deformation in the shear bands is significantly larger than expected for a single ECAP pass in a $90^{\circ}$ die, and this excessive straining is counter-balanced by considerably smaller amounts of straining in the matrix bands. Microstructural analysis by electron microscopy confirms this observation; even after a single pass, microstructural features typically associated with UFG materials are identified inside the shear bands.

Additional numerical analysis and novel experimental work was performed in order to understand which factors promote unstable plastic flow and shear banding during ECAP of this alloy. From a mechanical point of view, kinematic hardening and a high initial hardening rate combined with a low hardening rate at higher strains leads to the formation of distinct shear and matrix bands that agree well with our experimental observations. This kind of mechanical behavior is of course intricately linked to texture of the initial material. Finally, it was shown that the local stress state (and the stress gradient) at the inner die corner, which can be manipulated by modifying the corner radius geometry, has a pronounced effect on shear band widths.

We note in closing that the bulk laminated AA6060 material produced by ECAP, in addition to posing some scientifically challenging questions, exhibits interesting mechanical properties, with the UFG microstructures inside the shear bands significantly deviating from the mechanical behavior of the less deformed matrix bands. A more detailed analysis of both the local and global 
mechanical behavior of the bulk laminated materials will be addressed in a companion paper.

The authors would like to acknowledge fruitful discussions and a highly enjoyable scientific collaboration related to the simulation of strain partitioning with Tobias Horn, Christian Silbermann, and Jörn Ihlemann, all from the Solid Mechanics Group in Chemnitz. Early stages of this work have been funded by the German Research Foundation (Deutsche Forschungsgemeinschaft, DFG) through the Collaborative Research Center SFB 692.

\section{References}

1. A.H. Cottrell, B.A. Bilby, Proc. Phys. Soc. Sect. A. 62, 49-62 (1949)

2. A.H. Cottrell, Philos. Mag. J. Sci. 44, 829-832 (1953)

3. S.D. Antolovich, R.W. Armstrong, Prog. Mater. Sci. 59, 1-160 (2014)

4. J. Khalil-Allafi, B. Hasse, M. Klönne, M. Wagner, T. Pirling, W. Predki, W.W. Schmahl, Materwiss. Werksttech. 35, 280-283 (2004)

5. C. Elibol, M.F.-X. Wagner, Mater. Sci. Eng. A 643, 194-202 (2015)

6. M.F.-X. Wagner, G. Eggeler, Mech. Mater. 38, 1012-1025 (2006)

7. M.A. Meyers, Y.B. Xu, Q. Xue, M.T. PérezPrado, T.R. McNelley, Acta Mater. 51, 13071325 (2003)

8. M. Dolinski, D. Rittel, A. Dorogoy, J. Mech. Phys. Solids. 58, 1759-1775 (2010)

9. R.Z. Valiev, Y. Estrin, Z. Horita, T.G. Langdon, M.J. Zehetbauer, Y.T. Zhu, JOM 58, 33-39 (2006)

10. R. Valiev, Nat. Mater. 3, 511-516 (2004)

11. Y. Iwahashi, Z. Horita, M. Nemoto, T.G. Langdon, Acta Mater. 46, 3317-3331 (1998)

12. M. Gaspérini, C. Pinna, W. Swiatnicki, Acta Mater. 44, 4195-4208 (1996)

13. R. Lapovok, L.S. Tóth, A. Molinari, Y. Estrin, J. Mech. Phys. Solids 57, 122-136 (2009)

14. K. Hockauf, M.F.-X. Wagner, T. Halle, T. Niendorf, M. Hockauf, T. Lampke, Acta Mater. 80, 250-263 (2014)

15. P. Frint, M.F.-X. Wagner, Acta Mater. 176, 306317 (2019)

16. S. Frint, M. Hockauf, P. Frint, M.F.-X. Wagner, Mater. Des. 97, 502-511 (2016)

17. Y. Iwahashi, J. Wang, Z. Horita, M. Nemoto, T.G. Langdon, Scr. Mater. 35, 143-146 (1996)

18. P. Frint, M.F.-X. Wagner, S. Weber, S. Seipp, S. Frint, T. Lampke, J. Mater. Process. Technol. 239, 222-229 (2017)

19. I.J. Beyerlein, C.N. Tomé, Mater. Sci. Eng. A 380, 171-190 (2004)

20. I.J. Beyerlein, L.S. Tóth, Prog. Mater. Sci. 54,
427-510 (2009)

21. L.S. Tóth, Adv. Eng. Mater. 5, 308-316 (2003)

22. L.S. Tóth, R. Arrufat Massion, L. Germain, S.C. Baik, S. Suwas, Acta Mater. 52, 1885-1898 (2004)

23. A. Vinogradov, Y. Estrin, Prog. Mater. Sci. 95, 172-242 (2018)

24. T.A. Lebedkina, M.A. Lebyodkin, J.-P. Chateau, A. Jacques, S. Allain, Mater. Sci. Eng. A 519, 147-154 (2009)

25. H. Ovri, E.T. Lilleodden, Acta Mater. 89, 88-97 (2015)

26. H. Ovri, E.T. Lilleodden, Mater. Des. 125, 69-75 (2017)

27. A. Korbel, F. Dobrzanski, M. Richert, Acta Metall. 31, 293-298 (1983)

28. A. Korbel, V.S. Raghunathan, D. Teirlinck, W. Spitzig, O. Richmond, J.D. Embury, Acta Metall. 32, 511-519 (1984)

29. A. Korbel, M. Richert, Acta Metall. 33, 19711978 (1985)

30. A. Korbel, P. Martin, Acta Metall. 34, 1905-1909 (1986)

31. T.D. Horn, C.B. Silbermann, P. Frint, M.F.-X. Wagner, J. Ihlemann, Metals 8, 55 (2018)

[32] R.B. Figueiredo, I.P. Pinheiro, M.T.P. Aguilar, P.J. Modenesi, P.R. Cetlin, J. Mater. Process. Technol. 180, 30-36 (2006) 